Ponomarenko V., Ph. D.

Simon A., eng. 2 кат.,

K. Chiuriumov, Dr. Sci., professor

Astronomical Observatory of National Taras Shevchenko University of Kyiv

\title{
PARAMETERS OF DUST AND GAS IN THE COMMA OF THE COMETS C/2014 Q2 (LOVEJOY) I C/2013 US10 (CATALINA)
}

The observations and research of the long periodic comets C/2014 Q2 (Lovejoy) and C/2013 US10 (Catalina) by optical spectra with an average resolution $(N \Delta \Lambda \approx 1200)$ are presented. The spectra were obtained in February and December 2015 using the telescope $A Z T-14(D=0.48 m, F=7.7 m)$ and the spectrograph ASP-9 at the station Astronomical Observatory of Taras Shevchenko National University of Kyiv "Lisnyky". On the basis of obtained spectral material was carried the identification of spectral emission bands. Calculated some physical parameters of neutral gas cometary atmosphere and dusty cometary atmosphere. Built distribution of general and reflected energy along the slit of the spectrograph. Calculated flows, the number of molecules and gas productivity for basic molecular emissions, relative dust productivity.

В. Пономаренко, канд. физ.-мат. наук,

А. Симон, инженер 2 кат.,

К. Чурюмов, д-р физ.-мат. наук, проф.

Астрономическая обсерватория Киевского национального университета

имени Тараса Шевченко

\section{ПАРАМЕТРЫ ПЫЛИ И ГАЗА В КОМЕ КОМЕT C/2014 Q2 (LOVEJOY) И C/2013 US10 (CATALINA)}

Представлены результаты наблюдений и исследований долгопериодических комет C/2014 Q2 (Lovejoy) u C/2013 US10 (Catalina) на оcнове оптических спектров со средней разделительной способностью (N/A $\approx 1200)$. Спектры были получены в феврале и декабре 2015 года с помощью телескопа АЗТ-14 (D = 0.48 м, F = 7.7 м) и спектрографа АCП-9 на наблюдательной станции "Лесники" Астрономической обсерватории Киевского национального университета имени Тараса Шевченко. На основе полученного спектрального материали была проведена идентификация спектральных эмиссионных полосс. Найдены некоторые физические параметрв нейтральной газовой и пылевой кометных атмосфер. Построено распределение общего и отраженного потока энергии вдоль щели спектрографа. Вычислены потоки, количество молекул и газопродуктивность для основных молекулярных эмиссий, относительная пылепродуктивность.

УДК 521.95, 521.96, 52-14

И. Эглитис, канд. физз.-мат. наук,

М. Эглите, мл. науч. сотрудник Институт астрономии Латвийского университета, Рига В. Андрук, науч. сотрудник,

Л. Пакуляк, канд. физ.-мат. наук Главная астрономическая обсерватория НАН Украины, Киев

\section{U-ВЕЛИЧИНЫ ЗВЕЗД И ГАЛАКТИК ИЗ ОЦИФРОВАННЫХ АСТРОНЕГАТИВОВ ТЕЛЕСКОПА ШМИДТА В БАЛДОНЕ}

Выполнена оцифровка и обработка нескольких десятков пластинок из стеклотеки 1,2 м телескопа Шмидта в Балдоне, экспонированных в U полосе Джонсона. Для оцифровки использованы сканеры Epson Expression, обработка выполнена в пакете LINUX/MIDAS/RAMAFOT. Точность определения положений звезд Tychо2 составляет 0,1 ", точность построения характеристических кривых в U-полосе заключена в пределах 0,1-0,2 ${ }^{m}$.

Введение. Наблюдения в ультрафиолетовой (UV) полосе спектра в общем случае выполняются вблизи вспыхивающих или сверхновых звезд, на участках с активными ядрами галактик, в областях звездообразования и в полях со скоплениями. Особенно много UV данных в работах по исследованию переменных типа карликовых новых, квазаров и черных дыр [8; 9]. Однако, как следует из данных каталогов и публикаций VisieR Страсбургского центра астрономических данных, в целом UV наблюдений значительно меньше, чем, например, в B, V или R полосах.

Обсерватория в Балдоне Астрономического института Латвийского университета хранит коллекцию из 22 тысяч фотографических пластинок, полученных на 1,2 м телескопе Шмидта. Площадь неба, которую охватывает каждая из пластинок, составляет 19 квадратных градусов и содержит от 10 до 50 тысяч изображений небесных объектов. Масштаб изображений составляет 72 "/ мм.

Наблюдательный материал и его оцифровка. Архив телескопа Шмидта насчитывает 734 пластинки в 253 областях неба, отснятых в UV полосе, близкой к стандартной U Джонсона. При наблюдениях использовалась комбинация эмульсий ORWO ZU21 и ZU2 и фильтров UG1 и UFS3. Пластинки UV коллекции сканировались на сканерах серии EPSON EXPRESSION 10000XL и 11000XL. Предварительные исследования показали, что оптимальным разрешением для сканированных изображений является $1200 \mathrm{dpi} \mathrm{(1,81} \mathrm{"/пх),} \mathrm{дающее} \mathrm{практически} \mathrm{ту} \mathrm{же} \mathrm{точность,} \mathrm{что} \mathrm{и}$ сканы, полученные с более высоким разрешением 2400 dрі. При этом время, затраченное на обработку одной пластинки на высокоскоростном семиядерном компьютере, сокращалось в три раза. Кроме того, существенно уменьшался объем хранимой информации. Особенности поведения сканеров серии EPSON EXPRESSION и их влияние на точность астрометрических и фотометрических результатов исследовалось предварительно в работах [2; 3; 4; 5; 8; $11 ; 24 ; 25]$. Случайные ошибки сканеров для астронегативов телескопа Шмидта оказались равны 0,04 " и 0,015 m для положений и звездных величин соответственно.

Этапы обработки астронегативов. Все изображения были получены в формате .tiff и перед обработкой переведены в формат .fit. Стандартная процедура обработки изображений выполнялась в программном пакете Linux / MIDAS / ROMAFOT, расширенном дополнительными приложениями, разработанными в ГАО НАН Украины для обработки широкоугольных звездных полей. В качестве опорного использовался каталог Тусhо2. Процедура обработки отсканированных астронегативов протестирована и реализована ранее в серии работ $[6 ; 7 ; 9 ; 12 ; 13 ; 14 ; 17$; 18; 19; 26; 28] и состоит из следующих этапов: 
1. Обработка сканов в пакете MIDAS/ROMAFOT, получение прямоугольных координат X, Y и инструментальных звездных величин m зарегистрированных объектов.

2. Создание файлов опорных звезд из каталога Tycho2 для каждой пластинки.

3. Создание файлов для предварительной связи прямоугольной и экваториальной систем координат опорных звезд.

4. Исправление прямоугольных координат объектов за систематические ошибки сканера.

5. Редукция прямоугольных координат X, Y зарегистрированных объектов в систему экваториальных координат $\alpha, \delta$ каталога Тусho2.

6. Приведение инструментальных фотометрических величин объектов в систему фотоэлектрических Uре величин звезд системы Джонсона.

Более полная информация об этапах обработки оцифрованных пластинок содержится в работах [16; 20; 23]

Астрометрическая редукция в систему каталога Tycho2. Для всех сканов пластинок как на этапе диагностирования систематических ошибок сканера $\Delta \alpha$ и $\Delta \delta$, так и на этапах редукции прямоугольных координат X, Y объектов в систему экваториальных координат $\alpha$, $\delta$ каталога Tycho2 тангенциальные координаты $\xi$, $\eta$ вычислялись из решения уравнений методом наименьших квадратов по формулам вида (1):

$$
\begin{array}{ll}
\xi_{i}=a_{1}+a_{2} X_{i} f+a_{3} Y_{i} f+a_{4} R_{i} m_{i}+a_{5} f+\sum b_{I m} X_{i} Y_{i}^{m}, & (I=0 \div 6, m=0 \div 6, I+m=n, n=1 \div 6) \\
\eta_{i}=c_{1}+c_{2} X_{i} f+c_{3} Y_{i} f+c_{4} R_{i} m_{i}+c_{5} f+\sum d_{I m} X_{i} Y_{i}^{m}, & (I=0 \div 6, m=0 \div 6, I+m=n, n=1 \div 6)
\end{array}
$$

где $\mathrm{i}=1,2, \ldots \mathrm{N}$ - количество звезд каталога Тусhо2 на пластинке; $\mathrm{X}_{\mathrm{i}}, \mathrm{Y}_{\mathrm{i}}$ и $\mathrm{R}_{\mathrm{i}}$ - координаты и расстояние изображений звезд относительно центра пластинки; $m_{i}-$ инструментальные фотометрические величины звезд; $f$ - диаметры изображений звезд (FWHM); коэффициенты $\mathrm{a}_{2}, \mathrm{a}_{3}, \mathrm{a}_{4}$ и $\mathrm{c}_{2}, \mathrm{c}_{3}, \mathrm{c}_{4}$ описывают кому, коэффициенты $\mathrm{a}_{5}, \mathrm{c}_{5}-$ учитывают влияние уравнения блеска (вычислялись отдельно); коэффициенты полного полинома шестой степени (27 членов) $\mathrm{b}_{\mathrm{Im}}$ и $\mathrm{d}_{\mathrm{Im}}$ в обобщенном случае описывают аберрации оптики телескопа, отягощенные систематическими ошибками сканеров. Более подробно этапы исключения систематических ошибок сканеров изложены в работе [20].

Фотометрическая редукция в систему фотоэлектрических U-величин. Для калибровки характеристических кривых астронегативов, учета фотометрической ошибки поля и редукции инструментальных фротометрических величин m в систему фотоэлектрических Uре величин звезд системы Джонсона использовались данные из каталогов [10; 22; 27]. При построении характеристических кривых астронегативов использовалась фотометрическая информация для одной экспозиции. Аппроксимация характеристических кривых и получение фотографических U величин звезд для пластинок выполнена решением методом наименьших квадратов систем уравнений согласно формуле (2):

$$
\mathrm{U}_{\mathrm{i}}=\mathrm{e}_{1}+\mathrm{e}_{2} \mathrm{X}_{\mathrm{i}}+\mathrm{e}_{3} \mathrm{Y}_{\mathrm{i}}+\mathrm{e}_{4} \mathrm{R}_{\mathrm{i}}+\sum \mathrm{f}_{\mathrm{n}} \mathrm{m}_{\mathrm{i}}^{\mathrm{n}}, \quad(\mathrm{n}=1 \div 5)
$$

где $\mathrm{i}=1,2, \ldots \mathrm{N}$ - количество фотоэлектрических определений для стандартных звезд на пластинке; $\mathrm{X}_{\mathrm{i}}, \mathrm{Y}_{\mathrm{i}}$ и $\mathrm{R}_{\mathrm{i}}-$ координаты и расстояние изображений звезд относительно центра пластинки; $\mathrm{m}_{\mathrm{i}}-$ инструментальные фротометрические величины звезд; коэффициенты е $e_{2}, e_{3}, e_{4}$ ответственны за фотометрическое уравнение (фотометрическую ошибку) поля, а коэффициенты $\mathrm{f}_{\mathrm{n}}$ соответствуют функциональному описанию самого вида характеристических кривых.
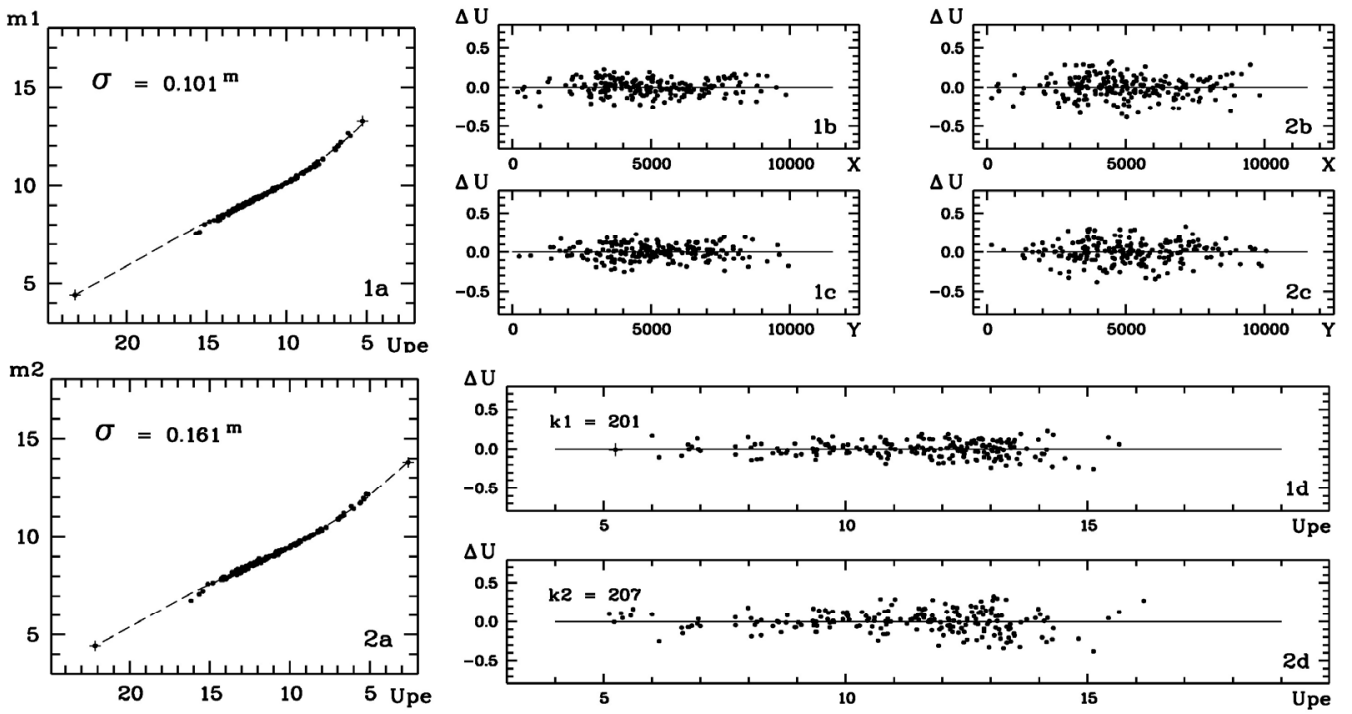

Рис. 1. Результаты фотометрической редукции для астронегативов с одной экспозицией

Данный вид формулы (2) выбран как оптимальный, позволяющий минимизировать фоотометрические ошибки редукции в систему фотоэлектрических стандартов Uре.

Результаты фотометрической редукции для астронегативов с одной экспозицией представлены на рис. 1. На рисунке панели 1а и 2а - характеристические кривые для двух перекрывающихся астронегативов, имеющих общие объекты, что позволяет оценить внутреннюю сходимость результатов обработки. m1 и m2 - инструментальные величины, Uре - фотоэлектрические величины. Указаны ошибки построения характеристических кривых $\sigma$. 
На панелях b,c,d приведены тренды разностей $\Delta U$ между вычисленными величинами и их фотоэлектрическими значениями Upe по координатам X, Y и звездным величинам Upe. На рисунке указано количество стандартных звезд k1 и k2 для обоих астронегативов.

После получения экваториальных координат $\alpha, \delta$ и звездных величин в системе U для каждой пластинки, было выполнено сравнение полученных результатов. Результаты сравнения в виде трендов разностей соответствующих величин представлены на левых панелях рис. 2. Здесь $\Delta \alpha, \Delta \delta$ - разности экваториальных координат, $\Delta U$ - разности звездных величин для общих объектов двух астронегативов. Тренды даны относительно координат X и $\mathrm{Y}$, Uре величин звезд и диаметров изображений f.
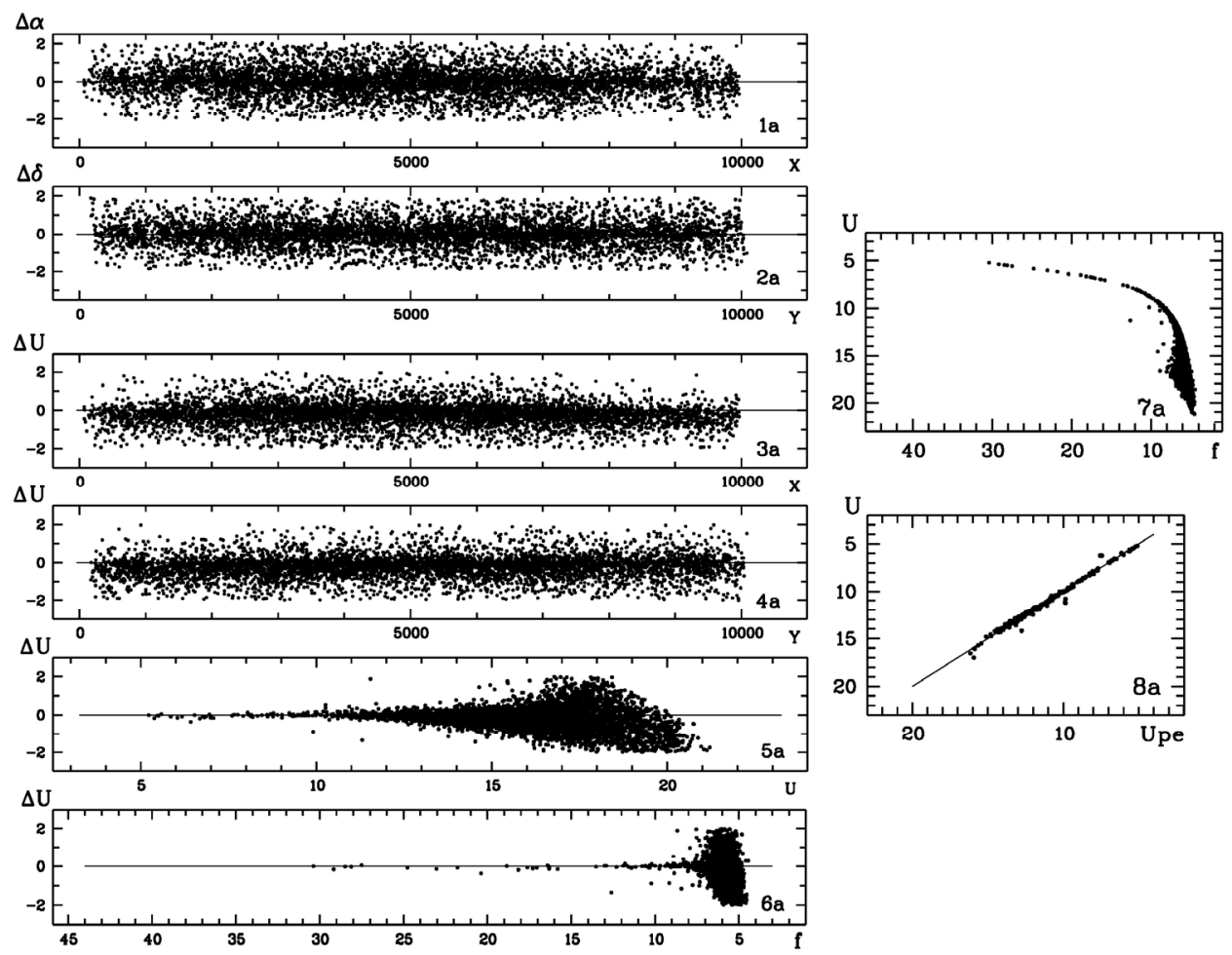

Рис. 2. Оценка внутренней сходимости результатов по общим для двух негативов объектам

На панелях 7а, 8а представлена связь U-величин с диаметрами изображений объектов и фотоэлектрическими стандартами Uре. Отметим, что для звезд с U<14 ${ }^{\mathrm{m}}$ среднеквадратическая величина разностей координат и звездных величин составляет $\sigma_{\alpha \delta}= \pm 0.14 "$ и $\sigma_{U}= \pm 0.12^{\mathrm{m}}$ соответственно.

Заключение. На настоящий момент по приведенной методике обработано около двух сотен астронегативов, экспонированных на 1,2 м телескопе Шмидта в Балдоне в UV полосе. Координаты звезд и галактик получены в системе каталога Tycho2, U-величины в системе фотоэлектрических стандартов. Работа по обработке астронегативов продолжается.

Полученный ранее по аналогичному наблюдательному материалу и описанной выше методике каталог положений и U-величин для 68784 звезд и галактик для 12 областей программы МЕГА [1; 15; 21] позволяет сделать прогноз относительно ожидаемой точности после завершения обработки всего наблюдательного материала. Внутренняя точность этого каталога для всех объектов составляет $\sigma_{\alpha \delta}= \pm 0.28$ " и $\sigma_{U}= \pm 0.20^{\mathrm{m}}$ (для звезд в интервале $U=8{ }^{\mathrm{m}}$ $-14^{\mathrm{m}}$ ошибки равны $\sigma_{\alpha \delta}= \pm 0.11$ " и $\sigma_{U}= \pm 0.09^{\mathrm{m}}$ ) для экваториальных координат и звездных величин соответственно. Сходимость координат с опорной системой Тусhо2 составляет $\sigma_{\alpha \delta}= \pm 0.06$ " (для 5814 звезд). Сходимость с фротоэлек-

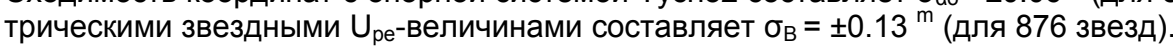

Список цитируемых источников

1. Андрук В. Н. Фотометрическая служба вблизи главного меридиана Галактики: наблюдения и создание каталога фотометрических стандартов звездных величин и цветов в системе UBVR // Кинематика и физика небес. тел. - 1996. - 12, № 4. - С. 60-73.

2. Андрук В. Н., Иванов Г. А., Погорельщев М. Т., Яценко А. И. Об использовании сканера для определения координат и фотометрии звёзд на пластинках программы ФОН // Кинематика и физика небес. тел. - 2005. - 21, № 5. - С. 396-400.

3. Андрук В., Пакуляк Л. Дослідження можливости використання сканерів Microtek для фотометрії зір // Журнал фрізичних досліджень. - 2007. - № 3. - C. 329-333.

4. Андрук В.М., Бутенко Г.З., Яценко А.И. Фотометрия пластинок, оцифрованных сканером MICROTEK SCANMAKER 9800XL TMA // Кинематика и физика небес. тел. - 2010. - 26. - № 3. - С. 75-81.

5. Андрук В.М., Пакуляк Л.К., Іжакевич О.М. та ін. Астрометрія платівок ДША, оцифрованих двома типами сканерів. Розділення зображень зір двох експозицій // Вісник КНУ ім.Т. Шевченка. Сер. "Астрономія" - 2012. - № 48. - С. 11-13.

6. Андрук В.Н., Головня В.В., Иванов Г.А. и др. Каталог экваториальных координат и В-величин звезд околополюсной области программы ФОН // Кинематика и физика небес. тел. - 2016. - 32. - № 1. - С. 56-69.

7. Андрук В.Н., Пакуляк Л.К., Головня В.В. и др. Каталог экваториальных координат и В-величин звезд программы ФОН // Кинематика и физика небес. тел. - 2016. - 32. - № 5 - С. 1--6.

8. Головня В., Андрук В., Яценко А. Астрометрія платівок ПША, оцифрованих сканером MICROTEK SCANMAKER 9800XL TMA // Журнал фрізичних досліджень. - 2010. - 14. - № 2. - С. 1-8. 
9. Казанцева Л.В., Шатохина С.В., Процюк Ю.И. и др. Результаты обработки оцифрованных фотографических наблюдений Плутона из коллекций УкрВО // Кинематика и физика небес. тел. - 2015. - 31. - № 1. - С. 58-80.

10. Корнилов В.Г., Волков И.М., Захаров А.И. и др. Каталог WBVR-величин ярких звезд Северного неба // Под ред. В.Г. Корнилова - М.: Изд-во Моск. ун-та, 1991. - 400 с. (Труды Гос. астрон. ин-та им. П.К. Штенберга, 63)

11. Муминов М.М., Каххаров Б.Б., Йулдошев К.Х. и др. Астрометрия тестовых пластинок, оцифрованных сканером Epson expression 10000XL в Acтрономическом институте АН Руз // Известия ГАО в Пулкове. - 2013. - 220. - С. 517-521.

12. Муминов М.М., Эгамбердиев Ш.А., Латыпов А.А. и др. Каталог экваториальных координат и В-величин звезд экваториальной зоны программы ФОН на основе обработки оцифрованных астронегативов Китабской обсерватории // Известия ГАО в Пулкове. - 2016. - 223. - С. 339-346.

13. Процюк Ю.И., Мартынов М.В., Мажаев А.Э. и др. Создание каталогов координат и собственных движений звезд с использованием совместной обработки архивных фотографических и современных ПЗС-наблюдений // Кинематика и физика небес. тел. - 2014. - 30. - № 6. - С. 54-65.

14. Яиенко А.И., Андрук В.Н., Головня В.В. и др. Результаты сканирования снимков 60-й зоны программы ФОН - методика редукции измерений, характеристика выходного каталога // Кинематика и физика небес. тел. - 2011. - 27. - № 5. - С. 49-59.

15. Andruk V.M., Kharchenko N.V., Schilbach E., Scholz R.-D. Photometric survey near the main Galactic Meridian. 1. Photoelectric stellar magnitudes and colours in the UBVR system // Astron. Nachr. - 1995. - 316, N 4. - P. 225-248.

16. Andruk V.M., Vidmachenko A.P., Ivashchenko Yu.M. Processing of CCD frames of images of star fields without the frame of a flat field using new software in program shell of MIDAS/ROMAFOT // Kinematics and Physics of Celectial Bodies. Suppl. - 2005. N5. - P. 413-416.

17. Andruk V.M., Golovnya V.V., Ivanov G.A. et. al. Compilation of catalog of stellar equatorial coordinates and B-magnitudes using UkrVO plate database // Odessa Astron. Publ. - 2014. - 27, N 1. - P. 53-54.

18. Andruk V.M., Pakuliak L.K., Golovnia V.V. et al. Catalog of pasitions and B-magnitudes of stars in the circumpolar region of Northen Sky Survey (FON) project // Odessa Astron. Publ. - 2015. - 28, N 2. - P. 192-195.

19. Andruk V.M., Relke H., Protsyuk Yu.l. et al. Comparision of zero zone catalogues of the FON program based on the Kyiv and Kitab observations // Odessa Astron. Publ. - 2015. - 28, N2.- P. 188-191.

20. Andruk V.M., Pakuliak L.K., Golovnya V.V. et al. // - 2015, arxiv.org/abs/1512.05535.

21. Eglitis I., Eglite M., Andruk V.M., Pakuliak L.K. U-magnitudes of stars and galaxies from the digitized astronegatives of Baldone Schmidt telescope // -Astroplate-2016. - 2016. - In press.

22. Mermilliod J.C. Homogeneous means in the UBV system. - Institut d'Astronomie. Universite de Lausanne, 1991.

23. Protsyuk Yu.I., Andruk V.N., Kazantseva L.V. Software for processing of digitized astronegatives from archives and Databases of Virtual Observatory // Odessa Astron. Publ., - 2014. - 27, N 1, - P. 59-60.

24. Protsyuk Yu.I., Andruk V.N., Muminov M.M. et al. Method for evaluating the astrometric and photometric charakteristics of commercial scanners in their application for the scientific purpose // Odessa Astron. Publ., - 2014. - 27, N1. - P. 61-62.

25. Protsyuk Yu.I., Kovylianska O.E., Protsyuk S.V., Andruk V.M. Results of processing of astronegatives with commercial scanner // Odessa Astron. Publ., $-2014,-27$, N 1, - P. 63-64.

26. Protsyuk Yu., Andruk V., Mazhaev A. et. al. Determination of proper motins of circumpolar stars by using images from UkrVO plate archives // Odessa Astron. Publ. - 2015. - 28, N 2. - P. 202-203.

27. Relke E., Protsyuk Yu., Andruk V. The compiled catalogue of photoelectric UBVR stellar magnitudes in the Tycho2 system // Odessa Astron. Publ., $-2015,28$, N 2, - P. 211-212.

28. Yizhakevich O.M., Andruk V.M., Pakuliak L.K. Catalog of astronomical positions of Saturns moons obtained by photographic observations at the MAO NASU in 1961-1991 // Odessa Astron. Publ., - 2015. - 28. N 2. - P. 213-216.

Надійшла до редколегії 09.07.16

І. Еглітіс, канд. фіз.-мат. наук,

М. Егліте, мол. наук. співроб.

Інститут астрономії Латвійського університету, Рига,

В. Андрук, наук. співроб., Л. Пакуляк, канд. фіз.-мат. наук

Головна астрономічна обсерваторія НАН України, Київ

\section{U-ВЕЛИЧИНИ ЗІР І ГАЛАКТИК 3 ОЦИФРОВАНИХ АСТРОНЕГАТИВІВ ТЕЛЕСКОПА ШМІДТА В БАЛДОНЕ}

Виконані оцифрування та обробка декількох десятків платівок зі склотеки телескопа 1,2 м Шмідта в Балдоне, проекспонованих в U смузі Джонсона. Для оцифрування використані сканери Epson Expression, обробка виконана в пакеті LINUX/MIDAS/RAMAFOT. Tочність визначення положень зір Тусhо2 складає 0,1", точність побудови характеристичних кривих в U-смузі знаходиться в межах 0,1-0,2

I. Eglitis, Dr. phys., M. Eglite, MSc.

Institute of Astronomy, University of Latvia, Riga

V. Andruk, Msc., L. Pakuliak, Ph. D.

Astronomical Observatory NASU, Ukraine, Kyiv

\section{U-MAGNITUDES OF STARS AND GALAXIES FROM DIGITIZED ASTRONOMIC NEGATIVES OBTAINED BY BALDONE SCHMIDT TELESCOPE}

Digitizing and processing of several dozen Johnson $U$ plates from the archive of $1.2 \mathrm{~m}$ Baldone Schmidt telescope were made. Scans were obtained using Epson Expression scanners. Image processing was made in the LINUX/MIDAS/ROMAFOT package. Obtained positional accuracy of Tycho2 stars is 0.1 ". The precision of the characteristic curves restoration is $0.1-0.2^{m}$.

удк 521.95

В. Андрук, науч. сотрудник, Л. Пакуляк, канд. физ.-мат. наук,

В. Головня, науч. сотрудник,

С. Шатохина, науч. сотрудник,

Е. Ижакевич, мл. науч. сотрудник, Главная астрономическая обсерватория НАН Украины, Киев, Ю. Процюк, канд. физ.-мат. наук НИИ "НАО", Николаев

\section{О КАТАЛОГЕ ЭКВАТОРИАЛЬНЫХ КООРДИНАТ И В-ВЕЛИЧИН ЗВЕЗД ПРОГРАММЫ ФОН}

В рамках работ по программе ФОН с использованием накопленных ресурсов ОЦА (Объединенный цифровой Архив) УкрВО в ГАО НАН Украины создан каталог экваториальных координат $\alpha$, $\delta$ и В-величин звезд для северного полушария неба (от -4 град до +90 град). Количество обработанных пластинок равно 2260. Оцифровка астронегативов осуществлялась с помощью сканеров Microtek ScanMaker 9800XL TMA и Epson Expression 10000XL, режим сканирования - 1200 dpi, размер большинства пластинок - 30 х 30 см или 13000 х 13000 пкл. Каталог содержит 19451751 звезд и га- 